quently and in small numbers and were usually components of the normal skin flora, such as lactobacilli and Staphylococcus epidermidis. In six of the patients, however, the dominant anaerobic bacterial flora contained other potential pathogens (Trichomonas vaginalis in one, Candida albicans in three, Staph aureus in one, and herpes simplex virus in one).

\section{Discussion}

Although few reports on anaerobic erosive balanoposthitis have been published the condition is not uncommon; however, it is probably rarely recognised. It was formerly regarded as a typical fusospirochaetal infection ${ }^{6}$ but clearly it may be caused by a variety of non-sporing anaerobes, although microorganisms that dominate the normal anaerobic bacterial flora of the oropharynx are frequently implicated. The microorganisms which cause anaerobic balanitis may be transmitted from the mouth by fingers contaminated with saliva or more commonly orogenital contact. The infection may also be acquired venereally from women with overt anaerobic vaginitis or from those whose normal vaginal flora is unusually dense.

Poor genital hygiene and phimosis predispose to anaerobic erosive balanitis; the infection does not occur in those who have been circumcised. In neglected cases anaerobic balanoposthitis may progress to gangrenous balanitis (or phagedaena), in which there is rapid destruction of the glans and, in some cases, of the entire shaft of the penis. Such advanced cases are not uncommon in some countries of the Third World.

Acute anaerobic erosive balanitis is readily diagnosed clinically by the three features referred to earlier and rapidly responds to metronidazole treatment $(200-400 \mathrm{mg}$ orally three or four times daily for five days).

\section{References}

${ }^{1}$ Waugh MA, Evans EGV, Nayyar KC, et al. Clotrimazole (Canesten) in the treatment of candidal balanitis in men. Br $\mathcal{F}$ Vener Dis 1978;54:184-6. Brook I. Group B beta-haemolytic streptococci causing balanitis. South Med f 1980;73:1095.

${ }^{3}$ Peutherer JF, Smith IW, Robertson DHH. Necrotising balanitis due to a generalised primary infection with herpes simplex virus type $2 . \mathrm{Br} f$ Vener Dis $1979 ; \mathbf{5 5}: 48-51$

Krupicz J, cited by Rook A, Wilkinson DS, Ebling FJG. Textbook of dermatology. Oxford: Blackwell, 1968.

${ }^{5}$ Corbus $\mathrm{BC}$, Harris FG. Erosive and gangrenous balanitis. The fourth venereal disease. $\mathcal{F} A M A 1909 ; 52: 1474-7$.

${ }^{6}$ Brams J, Pilot I, Davis DJ. Studies of fusiform bacilli and spirochetes. II Their occurence in normal preputial secretions and in erosive and gangrenous balanitis. 7 Infect Dis 1923;32:159-66.

\title{
Benzodiazepine hypnotics remain effective for 24 weeks
}

\author{
IAN OSWALD, CATHERINE FRENCH， KIRSTINE ADAM， JANET GILHAM
}

\begin{abstract}
Ninety-seven poor sleepers aged 40-68 years took capsules nightly for 32 weeks and made daily subjective ratings. The benzodiazepine hypnotics lormetazepam $2 \mathrm{mg}$ and nitrazepam $5 \mathrm{mg}$ appeared still to improve sleep after 24 weeks of intake when compared with continuous placebo intake. The sustained effectiveness was most evident in a significant shortening of the time taken to fall asleep in patients receiving lormetazepam. After withdrawal, rebound occurred, so that, for two to three weeks, sleep latency and the quality of sleep were significantly worse than baseline values. The impairment was maximal on the second night after withdrawal of lormetazepam and on the fourth night after withdrawal of nitrazepam.
\end{abstract}

It is concluded that benzodiazepines remain effective for at least 24 weeks but that a period of disturbed sleep may be expected after withdrawal.

\section{Introduction}

Patients often take hypnotic drugs for months or years, yet the Committee on the Review of Medicines" saw "little evidence

University Department of Psychiatry, Royal Edinburgh Hospital, Morningside Park, Edinburgh EH10 5HF

IAN OSWALD, MD, FRCPSYCH, professor

CATHERINE FRENCH, MB, CHB, research fellow

KIRSTINE ADAM, PHD, research fellow

JANET GILHAM, BSC, MRC research scholar that sedative hypnotics, including benzodiazepines, continue to be effective when used nightly in patients over long periods." The committee also pointed to withdrawal symptoms after prolonged intake, and, after small doses, we have found these symptoms to last two weeks or more. We compared the subjective effects of taking placebo or nitrazepam $5 \mathrm{mg}$ or lormetazepam $2 \mathrm{mg}$ nightly for 24 weeks.

\section{Methods}

We studied 100 volunteers (68 women and 32 men) aged 40-68 years who were poor sleepers. Through a chain of contacts we intimated that we required volunteers not currently taking sleeping pills or other central nervous system drugs, willing to help for 32 weeks, and with compatible work habits. Interviews with over 300 people, to eliminate good or average sleepers, left 100 who considered themselves to be poor sleepers and who entered the study after a general medical examination. They agreed to take no other hypnotic or central nervous system drugs during the study and were cautioned over driving, especially after alcohol.

The study was double blind, except that we knew that the subjects would be given a placebo during the initial and final four-week periods. The 100 subjects were divided into groups of four for distribution of the drugs. In any group of four two subjects received lormetazepam $2 \mathrm{mg}$, one nitrazepam $5 \mathrm{mg}$, and one placebo. The order of drugs within every four was randomised. Entry into the study was spread over six weeks. Capsules were taken at bedtime. Fifty subjects took a placebo for four weeks followed by lormetazepam $2 \mathrm{mg}$ for 24 weeks followed by placebo for four weeks; 25 took a placebo for four weeks, nitrazepam $5 \mathrm{mg}$ for 24 weeks, and placebo for four weeks; and 25 took a placebo throughout the 32 weeks.

Each subject attended for a medical check at weekly intervals for the first eight weeks and thereafter every two weeks. The frequent contacts were important in sustaining co-operation and helped to reduce drop-outs. 
When the code was finally broken we found that the three groups had been closely matched with regard to age, sex, marital state, and answers to a questionnaire about how refreshed they felt in the mornings. The group given lormetazepam comprised 19 men and 31 women, that given nitrazepam seven men and 18 women, and that given placebo six men and 19 women. The groups were also closely matched for social class, though 53 subjects fell into the Registrar General's class 3 and the remainder into class 1 or 2 . The method of finding the volunteers and the fact that they had to engage in daily introspection and describe their feelings through abstract concepts made a social-class bias inevitable.

Each morning, 20 minutes after rising, the subjects made a rating on a $100 \mathrm{~mm}$ visual analogue scale of how vigilant they felt (marvellously alert and energetic to awfully sleepy and lacklustre), how well they thought they had slept (worst possible to best ever), whether they had had any bad dreams (yes/no), and how long they thought it had taken them to fall asleep in minutes on a modified log scale. Each evening they indicated how anxious they had felt during the day, how lively, how alert in the afternoon, and how alert in the evening. In addition, they completed a checklist of 25 items to indicate whether they had been not at all, a little, or a lot bothered by constipation, itchy irritation, a tendency to cry, having to pass water frequently, feeling restless, feeling groggy, a lack of confidence and unsureness of self, indigestion, diarrhoea, hot and cold sensations, palpitations, clammy hands, feeling shaky or trembly, feeling tense, feeling dizzy or giddy, feeling lethargic or slow, lacking concentration, unclear or double vision, headache, nausea or sickness, stomach pains, a bad taste in the mouth, bad temper or feeling irritable, a dry mouth or throat, or slurred speech.

As the study was to give rise to 750000 items of information, we decided to sample the data obtained on the first four days of the fourth placebo week at the start of the study (pre values) and at selected four-day periods at later points-namely, the first four days of the first week of "active" treatment (defined to include continuing treatment with placebo; post 1 values), the first four days of the last week of active treatment (post 2 values), and the first four days of the first week of placebo treatment during withdrawal (post 3 values).

In examining the four-day periods data from each analogue scale were averaged for each individual for each four-day period. Thus for each person there was one mean for quality of sleep in the initial placebo period, two mean values during the active treatment period, and one immediately after this. The differences between the mean for the initial placebo period (the pre value) and each of the three other values was then obtained by subtraction to give three numerical values for each subject. These values were used to test null hypotheses that there would be no differences among the treatment groups at the selected times. The differences in the three groups conformed well in scatter to normal distributions.

The null hypotheses were examined by a simple analysis of variance with three groups-those taking lormetazepam, nitrazepam, and placebo throughout. When appropriate, individual comparisons were then made using a Scheffé test ${ }^{2}$ with the criterion of significance set at $\mathrm{p}<0.05$.

The data on bad dreams and the 25 items on the evening checklist during the four-day periods were examined by noting whether or not a subject had at least one positive score-that is, either "a little" or "a lot." Thus the maximum score for any subject in a four-day period was one.

A special analysis was made of quality of sleep and latency in the withdrawal period. The mean of the 28 baseline values for each subject was subtracted from each of the values for that subject during the first 10 days after the end of the active treatment period (days 198-207). The null hypothesis-that there would be no difference on any of these 10 days from the baseline mean-was then tested with a simple analysis of variance for the three groups. In cases where the null hypothesis was rejected Scheffé tests were carried out and, because we were looking for adverse effects, the criterion for the presence of a rebound was set at $\mathrm{p}<0 \cdot 10$.

\section{Results}

Quality of sleep-Lormetazepam and nitrazepam both improved the subjective quality of sleep, whereas placebo had no effect. Fig 1 shows this by five-point moving means of daily scores and suggests that withdrawal of both drugs caused subjective impairment of sleep. Analysis of variance and subsequent Scheffé tests showed that the improvement in the quality of sleep with nitrazepam and lormetazepam found during the first four days of drug intake (post 1 values) was significantly different from any change in those volunteers who took

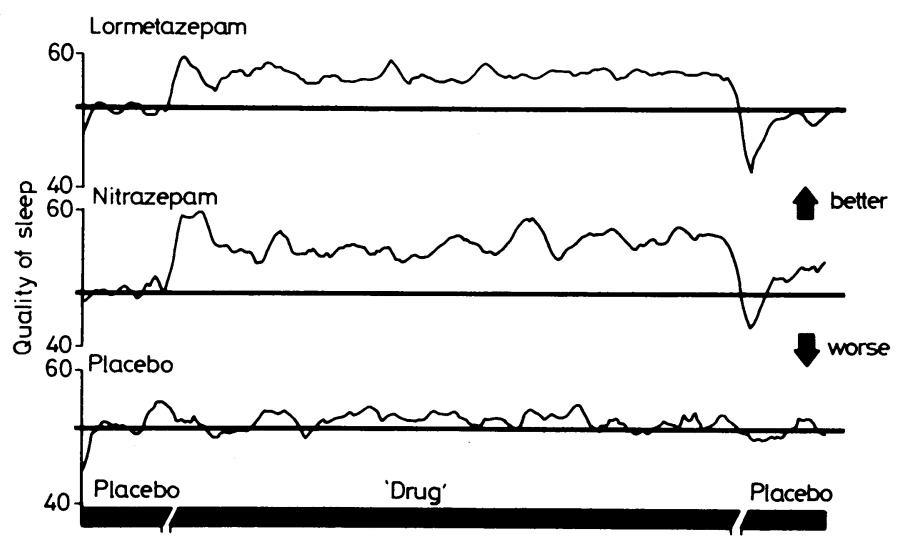

FIG 1-Daily self-ratings of quality of sleep during 32 weeks, smoothed and shown as five-point moving means. Compared with placebo, both drugs appeared to cause sustained improvement during the period of active treatment, with rebound worsening after withdrawal. (Horizontal lines represent baseline means.)

placebo throughout. There was no difference between the two drug groups. The difference between the value in the initial placebo period (pre value) and the post 2 value did not continue to be significant in either group when compared with placebo. Likewise, there were no significant differences between the placebo group and the two drug groups when post 3 minus pre values were compared. Although the preselected four-day periods thus did not show continued effectiveness of the drugs, the wider data displayed in fig 1 suggest a degree of sustained effectiveness for quality of sleep.

Sleep latency-Analysis of variance and Scheffé tests showed that in the shortening of sleep latency at post 1 the two drug groups were significantly different from the placebo group (fig 2). There was no significant difference between the two drug groups. The significant difference from placebo held again at post 2 for both drugs, thus suggesting sustained effectiveness during the 24 weeks of active treatment. Fig 2 shows a low point on the graph for nitrazepam corresponding with post 2 values, not consistently present, whereas the significant effect still present after taking lormetazepam for 24 weeks appeared to be consistent. There were no significant differences among the groups at post 3-that is, during withdrawal.

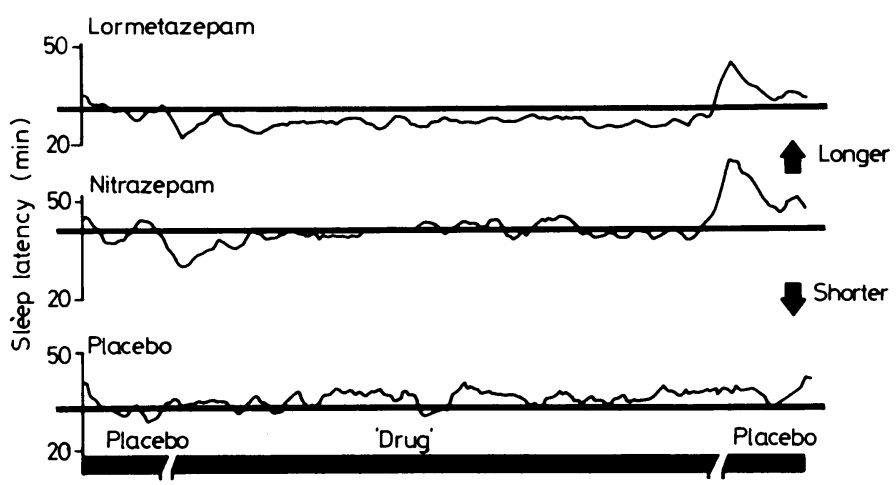

FIG 2-Self-ratings of how long it took to fall asleep during 32 weeks. Lormetazepam $2 \mathrm{mg}$ appeared to be associated with sustained shorter latency to sleep during the active treatment period. (Horizontal lines represent baseline means.)

Rebound-Figs 3 and 4 show unsmoothed mean data for individual days before and during the withdrawal period compared with the mean for the 28-day baseline period. Compared with placebo withdrawal of lormetazepam or nitrazepam caused a rebound in subjective quality of sleep and sleep latency. Inspection suggested that subjectively the rebound took two to three weeks to disappear. The peak of the rebound appeared to be on the fourth night after withdrawal of nitrazepam and on the second night after withdrawal of lormetazepam. Analysis of variance for the quality of sleep showed the presence of significant effects, and Scheffé tests showed that on the second and third nights after withdrawal of lormetazepam the difference from 
baseline values was significantly greater than that with placebo ( $p<0.05$ in each case) and this was also true for the mean of the first five nights after withdrawal. Likewise, for sleep latency the difference from baseline values was significantly greater on the second night after withdrawal of lormetazepam than that with placebo $(\mathrm{p}<0.05)$. Differences in quality of sleep after nitrazepam did not reach significance, but on the fourth and eighth nights after withdrawal of nitrazepam the difference in sleep latency from baseline values was significantly greater at the $p<0 \cdot 10$ criterion than the difference in the placebo group. Lormetazepam and nitrazepam differed from one another on the second night after withdrawal, when quality of sleep was worse in the group who had received lormetazepam $(\mathrm{p}<0.05)$, and again on the eighth night after withdrawal, when sleep latency was greater in the group who had received nitrazepam $(\mathrm{p}<0 \cdot 10)$.

Bad dreams, alertness, and anxiety-The rate at which bad dreams were reported among the 25 subjects given nitrazepam and the 25 who received placebo throughout was low and did not change. Among the 47 subjects who received lormetazepam and who completed the whole study, reporting of bad dreams fell during the two four-day periods monitored during active treatment with a return to the baseline value during withdrawal (table). No significant effects on ratings of alertness at any time of day were found for either drug

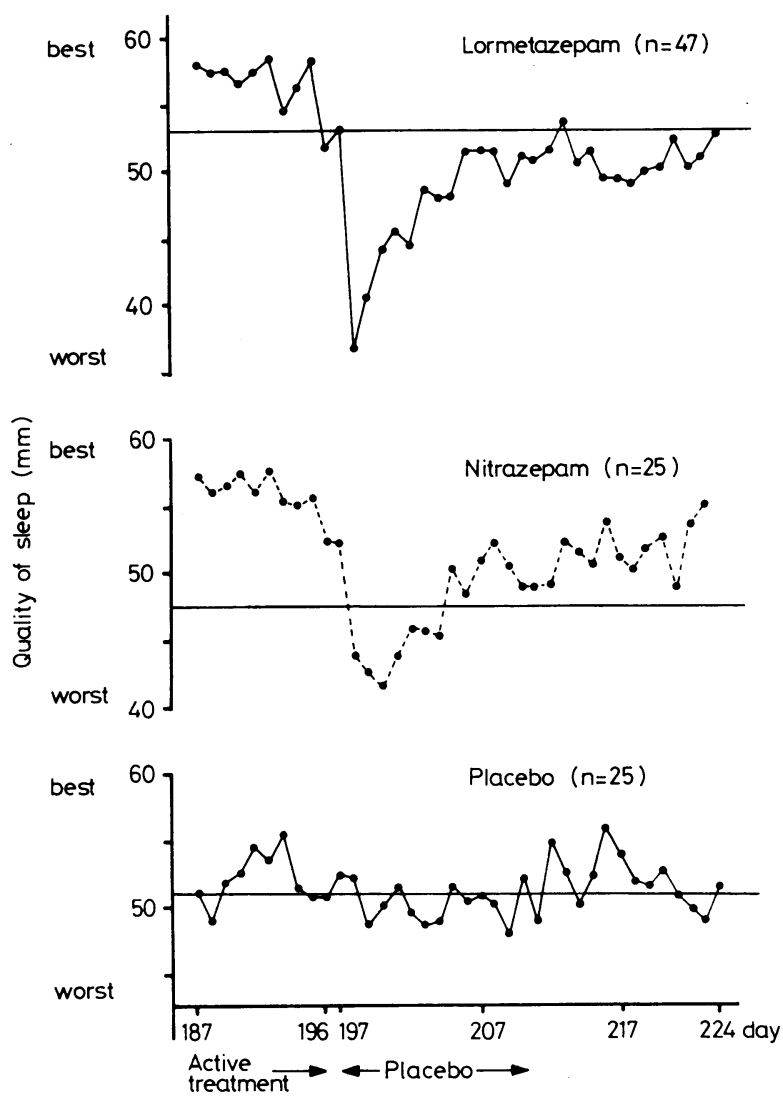

FIG 3-Rebound worsening of quality of sleep after withdrawal of both active drugs. (Horizontal lines represent baseline means.)

compared with placebo, though ratings of alertness and energy suggested mild impairment during the first few days after withdrawal of both drugs, consistent with ratings of poorer sleep. No effect on anxiety ratings emerged.

The 25-item checklist-Individual side effects were examined during the four-day periods, particular attention being paid to dizziness or giddiness, headaches, lethargy, or feeling slow, bad tempered, or irritable. Neither drug caused these side effects to be reported. A total score for all variables for each four-week period of the study was computed, and no suggestion of any increase in symptoms was found during active treatment.

Medical examination and termination of study-No change was found during the final month of active treatment in results of a general medical examination, liver function, blood tests, electrocardiography, and chest $x$-ray films, though there was a small fall in body weight after nitrazepam and lormetazepam. ${ }^{3}$ Three volunteers dropped out of the study. The first was a tense, anxious woman of 50 , who dropped out on the 57 th day, after three days of symptoms of influenza (widespread at that time) and four weeks after starting lormetazepam. A man of 51 dropped out on the 96 th day because his wife had to have a kidney operation. A woman of 42 left from day 166, when her husband $\overparen{\supset}$ died. These drop-outs were from the group given lormetazepam, but they left for reasons that cannot be attributed to the medication.
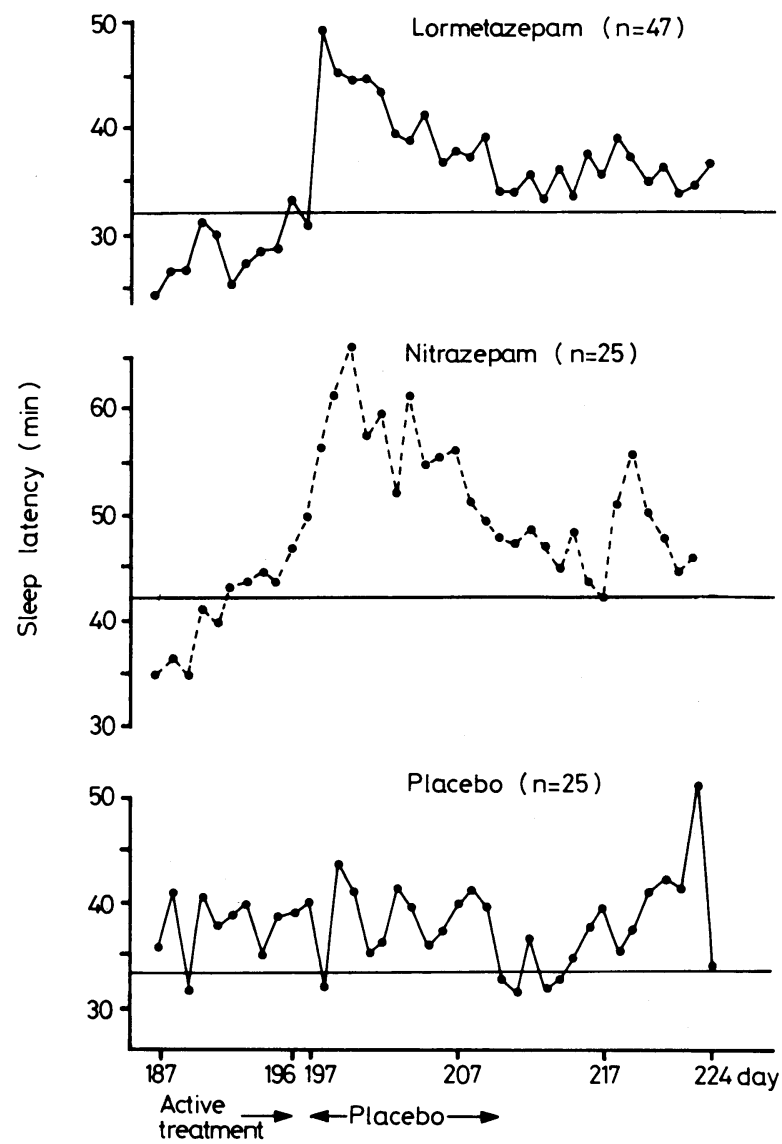

FIG 4-Rebound increase in time taken to fall asleep after withdrawal of both drugs. (Horizontal lines represent baseline means.)

Numbers of subjects reporting bad dreams

\begin{tabular}{lccc}
\hline $\begin{array}{c}\text { Time of } \\
\text { assessment }\end{array}$ & $\begin{array}{c}\text { Lormetazepam } \\
(\mathrm{n}=47)\end{array}$ & $\begin{array}{c}\text { Nitrazepam } \\
(\mathrm{n}=25)\end{array}$ & $\begin{array}{c}\text { Placebo } \\
(\mathrm{n}=25)\end{array}$ \\
\hline Pre & 11 & 2 & 5 \\
Post 1 & 2 & 1 & 3 \\
Post 2 & 1 & 2 & 3 \\
Post 3 & 11 & 1 & 3 \\
\hline
\end{tabular}

\section{Discussion}

Benzodiazepine hypnotics thus are effective from the first and retain some effectiveness after 24 weeks of nightly use, as seenco most clearly in the sustained reduction of sleep latency in $\mathbb{D}$ subjects taking lormetazepam $2 \mathrm{mg}$. The data on quality of $\stackrel{\mathbb{C}}{+}$ sleep, though appearing to show some improvement, no longer 0 reached the criterion of significance after 24 weeks, and because of this and the rebound after withdrawal some tolerance may be inferred.

Using electrophysiological measures we previously showed effectiveness of nitrazepam $5 \mathrm{mg}$ in the eighth week and then rebound insomnia on withdrawal. ${ }^{4}$ Using the same measures, rebound abnormalities of sleep have been seen to last four to five weeks after nitrazepam ${ }^{5}$ and other drugs, ${ }^{6}$ but in this study 
we quantified a time course of subjective insomnia and found its duration again to be measured more in weeks than in days. The duration is consistent with the two to four weeks of anxiety after withdrawal of benzodiazepines. ${ }^{78}$

Drugs that are more rapidly eliminated give rise to an earlier rebound, ${ }^{6}$ which would explain why lormetazepam (with a half life of only 10 hours $^{9}$ ) gave rise to a peak of rebound on the second night after withdrawal, whereas nitrazepam (with a half life of active metabolites of about 30 hours ${ }^{10}$ ) gave a peak on the fourth night. This interpretation is consistent with electroencephalographic signs of persistence of nitrazepam as late as three nights after withdrawal of a nightly dose of $15 \mathrm{mg} .^{5}$

Our findings suggest that it is desirable to prescribe benzodiazepines when an eventual reduction in life's problems is foreseen, with the aim of stopping the drugs coincident with that reduction; the patient should be told that a period of disturbed sleep may be expected after withdrawal.

We thank Mr K Fichte, Dr Susan Allen, Dr H Ott, Dr W Herrmann, and Schering AG.

\section{References}

${ }^{1}$ Committee on the Review of Medicines. Systematic review of the benzodiazepines. $\mathrm{Br}$ Med $\mathcal{F}$ 1980;280:910-2.

${ }^{2}$ Edwards AL. Statistical methods. New York: Holt, Rinehart and Winston, 1967:265-73.

${ }^{3}$ Oswald I, Adam K. Benzodiazepines cause small loss of body weight. Br Med f 1980;281:1039-40.

${ }^{4}$ Adam K, Adamson L, Březinová V, Hunter WM, Oswald I. Nitrazepam: lastingly effective but trouble on withdrawal. $\mathrm{Br}$ Med $\mathcal{F} 1976$; : 1558-60.

${ }^{5}$ Oswald I, Priest RG. Five weeks to escape the sleeping-pill habit. $\mathrm{Br} \mathrm{Med} \mathcal{F}$ 1965 ;ii:1093-5.

${ }^{6}$ Oswald I. Drug research and human sleep. Annual Review of Pharmacology $1973 ; 53: 243-52$.

${ }^{7}$ Allen S, Oswald I. Anxiety and sleep after fosazepam. Br 7 Clin Pharmacol $1976 ; 3: 165-8$

${ }^{8}$ Petursson $\mathrm{H}$, Lader $\mathrm{MH}$. Withdrawal from long-term benzodiazepine treatment. Br Med f $1981 ; 283: 643-5$.

${ }^{9}$ Hümpel M, Illi V, Milius W, Wendt H, Kurowski M. The pharmacokinetics and biotransformation of the new benzodiazepine lormetazepam in humans. Eur 7 Drug Metab Pharmacokinet 1979;4:237-43.

${ }^{10}$ Breimer DD, Bracht H, de Boer AG. Plasma level profile of nitrazepam following oral administration. Br f Clin Pharmacol 1977;4:709-11.

(Accepted 9 December 1981)

\title{
Controlled trial of slow-release aminophylline in childhood asthma: are short-term trials valid?
}

\author{
NICOLA WILSON, MICHAEL SILVERMAN
}

\begin{abstract}
Slow-release aminophylline, although widely used for the prophylaxis of childhood asthma, has had only limited formal assessment. A four-month double-blind cross-over trial of slow-release aminophylline $(14 \mathrm{mg} / \mathrm{kg}$ twice daily) was carried out in 24 children with perennial asthma. Satisfactory serum theophylline concentrations were obtained in 17 children, with few side effects. There was a significant improvement in mild daytime and night-time symptoms. The incidence of more severe symptoms was unaffected. Treatment did not improve the mean peak expiratory flow or reduce the incidence of use of bronchodilators.

It is concluded that slow-release aminophylline has a place in the prophylaxis of perennial childhood asthma but is unsuitable for children who suffer from severe attacks. The cross-over trial design has severe limitations.
\end{abstract}

\section{Introduction}

In recent years it has become common practice to treat children with moderately severe perennial asthma with continuous prophylaxis. Methylxanthines have been widely used in this way, particularly in the USA, where they are often the first line of treatment. Sustained-release oral preparations have the important advantage that only twice-daily administration is needed to give fairly stable serum theophylline concentrations over 24 hours. ${ }^{1}$ The clinical value in chronic childhood asthma

Department of Paediatrics and Neonatal Medicine, Institute of Child Health, Hammersmith Hospital, London W12 0HS

NICOLA WILSON, MB, DCH, honorary clinical lecture MICHAEL SILVERMAN, MD, MRCP, senior lecturer of conventional treatment with oral theophylline has been shown in controlled clinical trials. ${ }^{23}$ We designed the present study to show the value of an oral preparation of slow-release aminophylline in a group of children considered to need continuous prophylactic treatment for their asthma.

\section{Methods}

Trial design-The study was in the form of a double-blind crossover trial with active slow-release aminophylline and placebo given in random order, each for an eight-week period. A run-up period was not obligatory, although most patients underwent at least one month of close monitoring before entry to establish their need for continuous prophylaxis. There was no break between the first and second eightweek periods. Patients were instructed to take their trial drugs after food, twice daily, with a 12-hour interval in between. The slowrelease aminophylline (Phyllocontin, Napp Laboratories) was prescribed as a combination of $100 \mathrm{mg}$ and $225 \mathrm{mg}$ tablets to give a dose of about $14 \mathrm{mg} / \mathrm{kg}$. An indistinguishable placebo preparation was given during the control period. Additional beta-agonists were taken as required.

Patient selection-Children of school age who were able to use a peak flow meter and to swallow tablets and whose parents could reliably fill in a daily record card were selected if they were considered to require continuous treatment because of frequent symptoms (occurring on at least 10 days each month) or because they were already taking some form of daily non-steroid prophylactic treatment. Parental consent was obtained and the project approved by the research ethics committee of the hospital.

Patient evaluation-The children were seen every four weeks during the 16-week study. Blood was taken for estimation of serum theophylline concentration ${ }^{4}$ at least once during each drug period and any side effects noted. At home symptoms (cough and wheeze separately recorded for day and night) and twice-daily measurements of peak flow (the best of three readings using a Wright Minimeter, Airmed) were recorded on the daily record card (diary). Patients also recorded all drugs taken during the trial, including extra bronchodilators.

Analysis-The data were analysed in the light of guidelines given by Hills and Armitage ${ }^{5}$ for this type of trial. The results in the two 